\title{
New Interpretative Models for the Study of Urban Space
}

\author{
Fabio Bianconi \\ Marco Filippucci \\ Marco Seccaroni
}

\section{Abstract}

The research project aims to acquire data on the impact of an environment on humans. The process is based on the use of the EEG helmet synchronised to GPS: the EEG helmet is a device that non-invasively records 14 channels of the human brain through electrodes and, through an algorithm, transforms them into cognitive states in real time. Starting from this raw data, through the 'circumplex model', cognitive states can be transformed into emotions with the aim of showing what people on average feel in a given urban space. The data, which are linked through GPS to a position in space, are empirically recorded with statistically significant samples from which emotions are reprojected onto the mesh of the digitally reconstructed environment through a process of photomodelling.

Keywords

perception, EEG, GPS, representation, emotions.

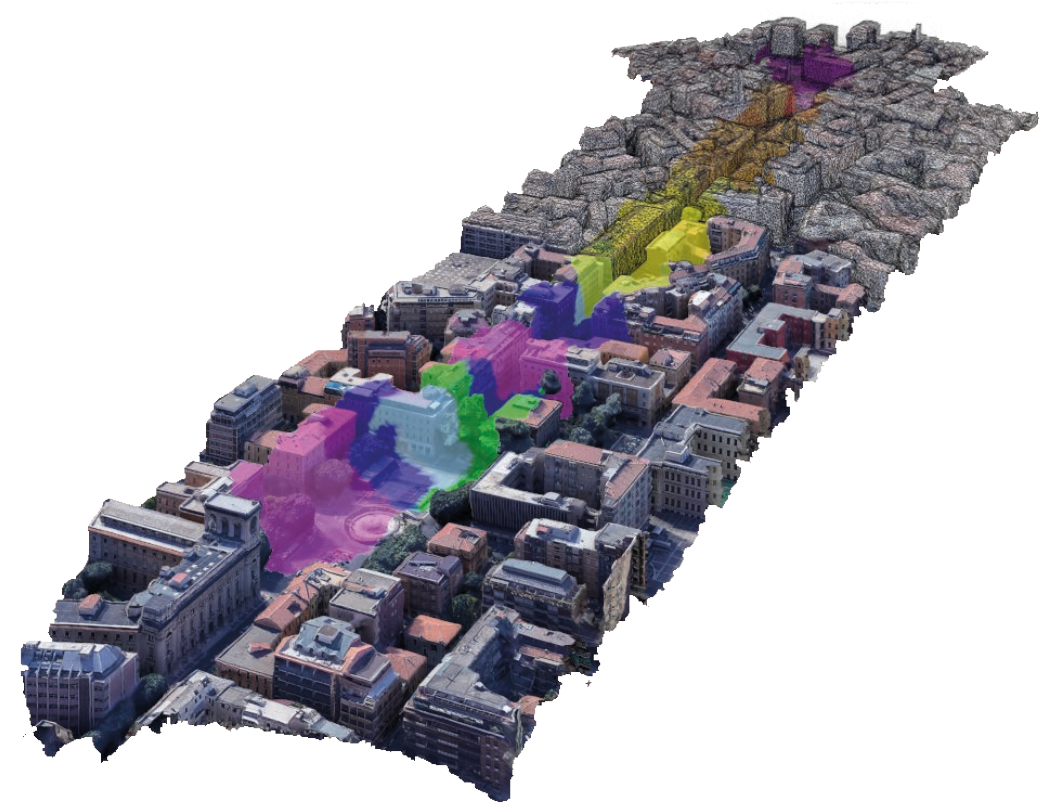




\section{Introduction}

If the theme of landscape is of great topicality, it is perhaps because, in its ethereal form, it represents the best contemporary utopia that is content to classify and circumscribe the problem of the relationship between man and the world around him, with the value of images, but without seeking solutions: by amplifying the distance between reality and its ideal model, there is often the risk of hiding the cultural and instrumental deficiency in the interpretation and representation of current phenomena and the strong current impediments in the construction of contemporary landscapes. This concept does not correspond with the concept of place, territory, environment, panorama [Bianconi \& Filippucci 2019], but as the European Convention formalises, "Landscape" means an area, as perceived by people, whose character is the result of the action and interaction of natural and/or human factors. The idea of landscape is therefore a cultural process, closely linked to perception [Arnheim 1986], to vision [Kepes, Rossi Chiaia 1990] and to those elements that structure the processes of identity construction.

Man, immersed in the built environment, is very often unaware of the influences it exerts on him. Generally, people gravitate towards spaces that may not be psychologically stimulating, but through habit tend to prefer a usual and known, albeit imperfect, pattern, rather than other configurations that would bring greater benefits from the point of view of psychophysical well-being and therefore identity. Even though most of our experiences in our surroundings do not take place consciously. In general, however, there is no such thing as a 'neutral' environment in an urban context: from the point of view of health, the man-made environment necessarily brings benefits or discomfort, contributing in the latter case to the onset of psychophysical illnesses. The built environment either helps or hurts [Goldhagen 2017], but design cannot be directly aimed at the health of citizens [Millennium Ecosystem Assessment 2005] and their well-being [Bechtel, Churchman 2002] if there is no methodology to analyse and evaluate the effects of solutions, then considering the social dimension of the individual's life [Schram-Bijkerk et al. 20।8].

\section{Research Themes}

The research aims to study the survey of landscape qualities [Bianconi, Filippucci 20 I 8]. The innovative aspects that characterize the research can be identified in the digital path developed aimed at the construction of interpretative tools of the relationship between the environment and its impacts, represented on the space. The analysis of people's behaviour in public space is developed using advanced digital tools and biosensors such as the EEG helmet. This makes it possible to define analytical and scientific data on feelings, by interpreting them it is possible to interpret the essential elements that influence well-being through the emotional state. The neuro-headset data makes it possible to understand which part of the cerebral cortex is affected by certain signals when immersed in an environment, thus providing interpretable data that is also useful for hypothesising solutions in compromised urban contexts. The EEG helmet is a device that non-invasively records 14 channels of the human brain through electrodes and, through an algorithm, transforms them into cognitive states in real time [Kim et al. 20।8]. This is functional for behaviour analysis [Aspinall et al. 20।5; Chynal et al. 2016; Tachi 2012; Yadava et al. 2017]: in fact, the raw data collected on brain impulses are interpreted by algorithms and transformed into six cognitive states (valence, arousal, stress, meditation, focus, engagement) [Badcock et al. 20 I5; Kotowski et al. 20 I8]. In the developed pathway, the combined use of the data is achieved through the circomplex model (fig. 4) [Posner et al. 2008] that allows emotions to be calculated using only the cognitive states of valence and arousal [Yik et al. $20 \mathrm{I} \mathrm{I}$ ].With the valence value as the first polar coordinate and the arousal value as the second polar coordinate, it is possible to obtain a point within the circumplex model that represents the emotion of the observer. These points are associated with a unique colour vector for each combination, transcribed in RGB colour space. In addition to the EEG data, the geographical position is acquired in real time at a regular interval of one second; thanks to the timestamp, it was possible to synchronize the GPS and EEG data. 
The experimental route leads to the collection of data on the position and cognitive states of several testers. It was therefore necessary to find a way to compare the emotions of different testers according to their spatial position. By means of an algorithm developed in the visual script language Grasshopper for the Nurbs environment of Rhinoceros, it is possible to import CSV data of the experiments containing synchronised coordinates and EEG data and identify at which positions the observations were taken from contiguous and thus comparable points. The developed algorithm [Bianconi et al. 2019] [Bianconi et al. 2020] then identifies the comparable recordings and calculates the average of the EEG and GPS values belonging to the same cell; in this way the average of the observers in space is obtained. The representation of the data is possible using as a base a mesh model of the territory (DEM) under analysis; the model is obtained through a process of photomodelling via google earth pro photos with $3 \mathrm{~d}$ buildings enabled. An attribute is added to the mesh base at each vertex, which corresponds to an RGB vector representing the average value in the circumscribed model of the sensation felt by the observers. Finally, the faces of the mesh are coloured by interpolating the vertex colours in a linear fashion. The representation thus obtained has a double objective: to store the instrumental data and to represent them in a comprehensible way (figs. 2-3).

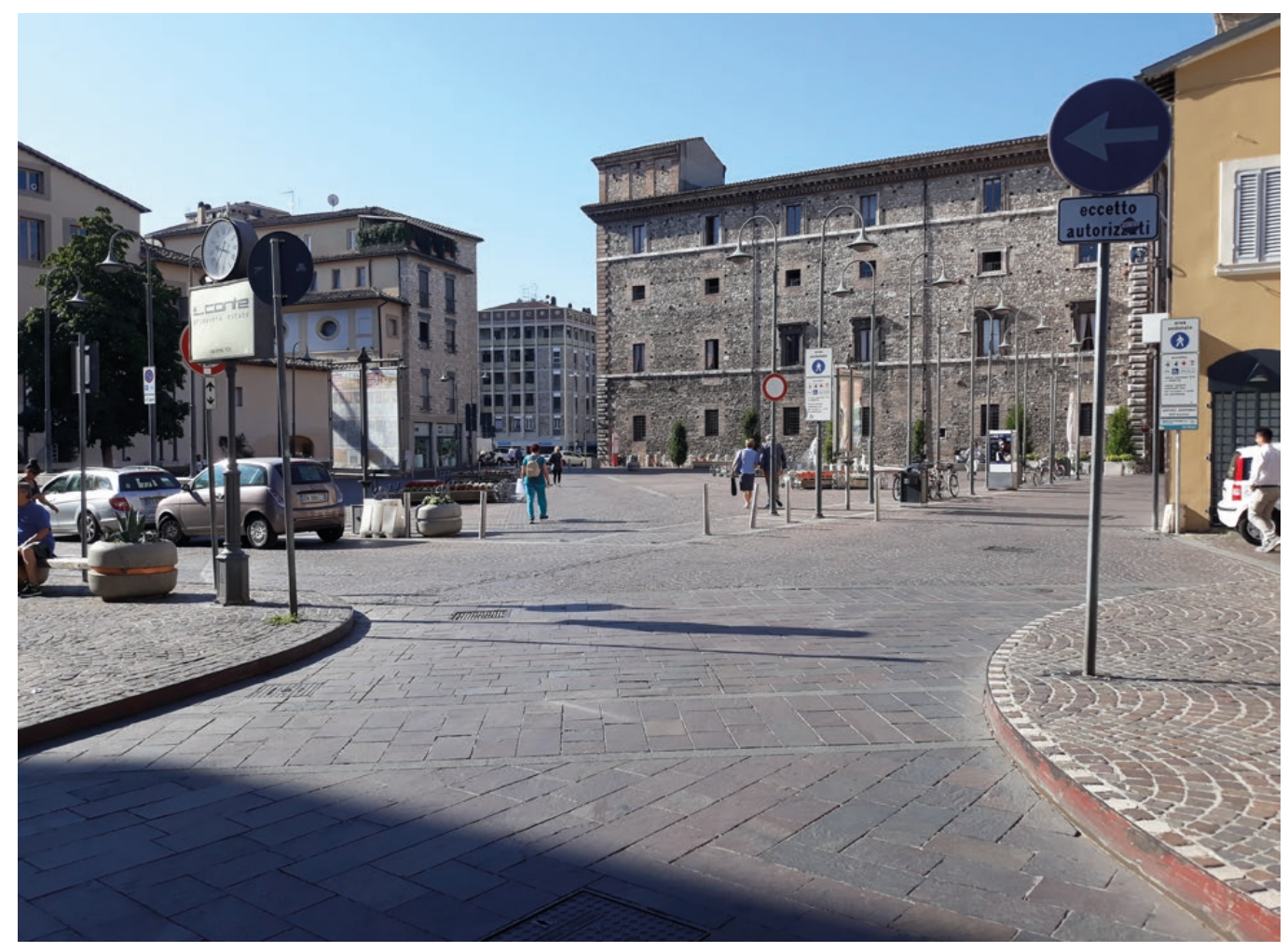

\section{The Case Study: Terni City Centre}

An illustrative case that shows the value of the analyses are the studies carried out in a portion of the historic centre of Terni that from Piazza Tacito, across the Corso, reaches Palazzo Spada (fig. I). The area under examination constitutes a section of considerable interest because within it there are many peculiarities. In an extremely circumscribed space there is a pedestrian area, intersections with roads, a square with a recent arrangement of street furniture and a square with an important Renaissance palace. And it is precisely the way in which these elements influence the emotional state of the user that is the subject of the analysis in question.

From the data acquired and processed it emerges that the area can be divided into four zones according to the emotions aroused in the observers. At the starting point, on average, 
a feeling of strong stress emerges due to the area highly congested with car traffic; continuing, the pedestrian area represents an area that oscillates between serenity and calmness except near the intersections with the driveways. In the final part of Corso Tacito a gradual transition from a state of calm to a high degree of stress in the vicinity of Palazzo Spada is clearly visible. This analysis shows that the pedestrian area of the city's main street is perceived as a comfortable space for users, but the fact that it is located between two highly trafficked squares limits its potential.

\section{Conclusions}

The proposal aims to define methodologies for data collection and interdisciplinary interpretative criteria to understand the impact of the environment on man, with the implicit objective of being able to direct design choices to build places that create wellbeing.

With an interdisciplinary approach and through the integration of multiple devices for detecting perception, it is possible to analyse what people feel and understand how the environment implicitly influences their emotional state and which places and conditions promote wellbeing.

Digital becomes a true Computer-aided Design tool, with digital data used not only to show the visible but also to make visible what is implicit in the relationship between man and the environment. The aim is thus to conform an innovative methodology that supports an operational verification of the meaning of places, which analytically focuses on emotions and meanings and can be used as a strategy for studying not only the territory and the environment, but also the landscape, understood as the result of the perception process. The creation of new data and their representation are the basis of new methodologies of investigation through the use of Al for the design and optimization of urban spaces.

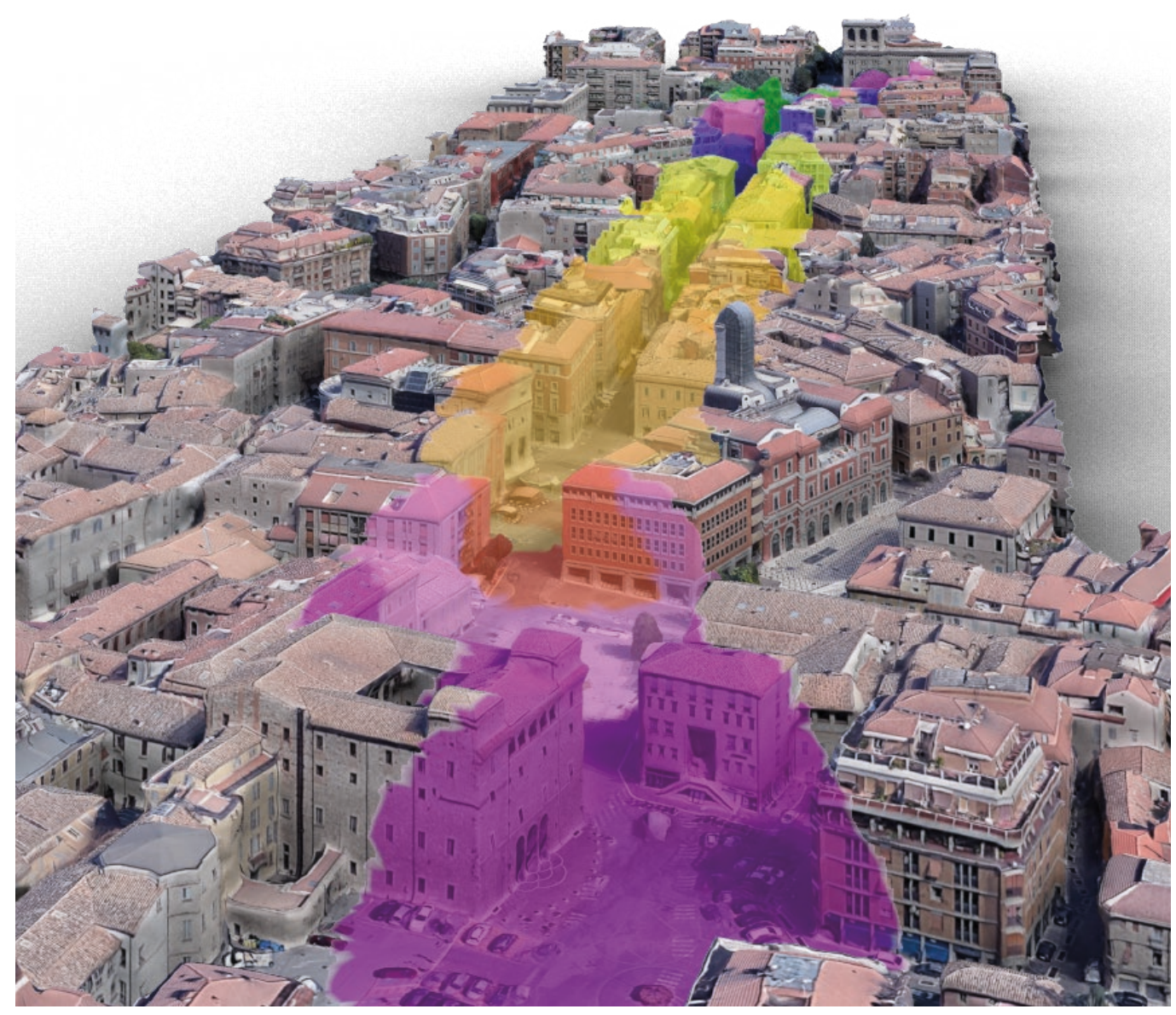


Fig. 3. In the perspective section, one can see the division of the average emotions felt during the experiment.
Fig. 4. Via the circomplex model is to represent through the colors the emotions.
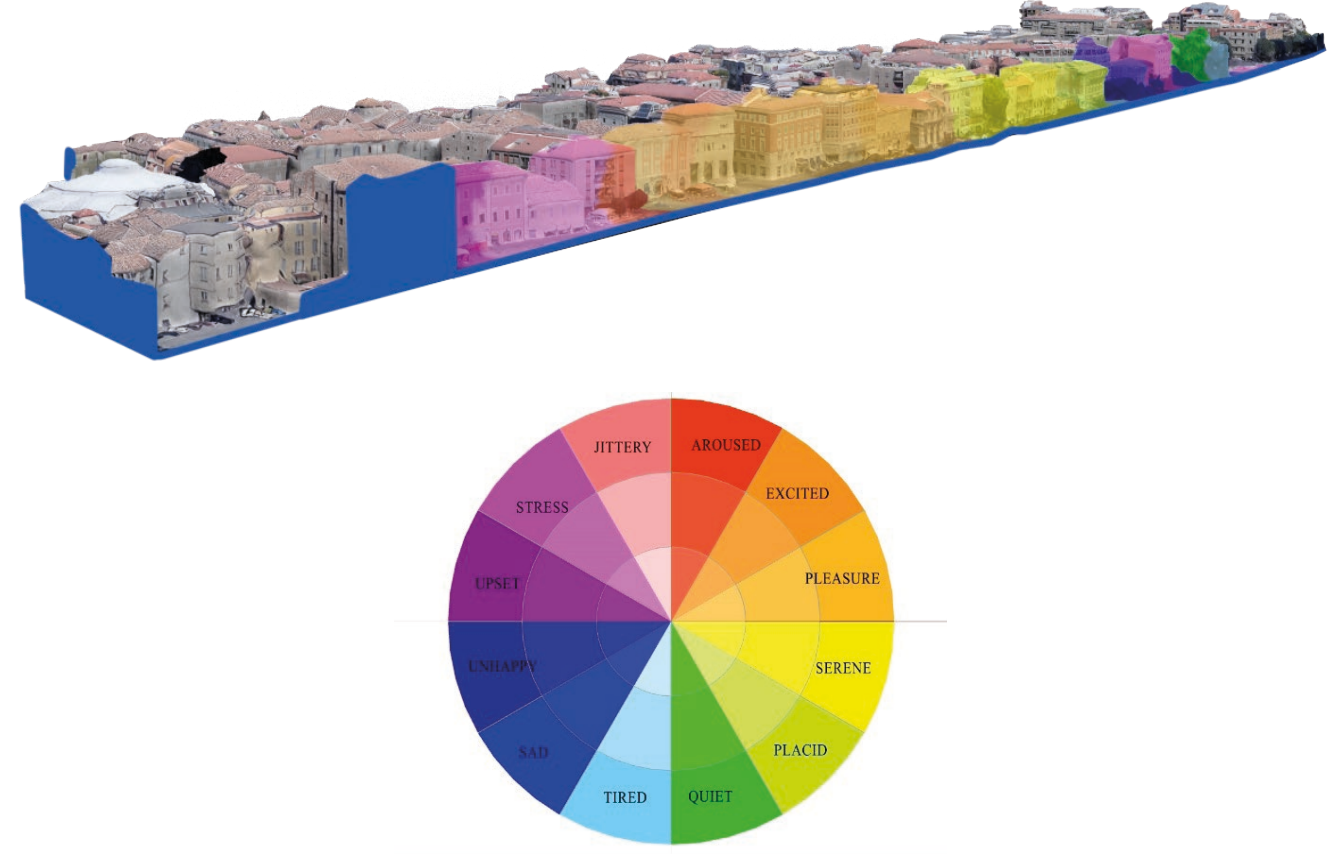

\section{References}

Arnheim Rudolf (1986). New essays on the psychology of art. Berkeley: University of California Press.

Aspinall Peter, Mavros Panagiotis, Coyne Richard, Roe Jenny (20I5). The urban brain: Analysing outdoor physical activity with mobile EEG. In British Journal of Sports Medicine, 49(4), pp. 272-276.

Badcock Nicholas A., Preece Kathryn A., de Wit Bianca, Glenn Katharine, Fieder Nora, Thie Johnson, McArthur Genevieve (20 I5). Validation of the Emotiv EPOC EEG system for research quality auditory event-related potentials in children. In Peer], 3, e907.

Bechtel Robert B., Churchman Arzah (2002). Handbook of environmental psychology. New York: J.Wiley \& Sons.

Bianconi Fabio, Filippucci Marco (eds.) (20 8). II prossimo paesaggio. Realtà, rappresentazione, progetto. Roma: Gangemi.

Bianconi Fabio, Filippucci Marco (2019). Landscape Lab. Drawing, Perception and Design for the Next Landscape Models. Cham: Springer.

Chynal Piotr, Sobecki Janusz, Rymarz Michal, Kilijanska Barbara (20 I 6). Shopping behaviour analysis using eyetracking and EEG. In Proceedings - 9th International Conference on Human System Interactions, HSI, pp. 458-464

Goldhagen Sarah Williams (2017). Welcome to Your World. How the Built Environment Shapes Our Lives. New York: Harper Collins. Kepes György, Rossi Chiaia Franca (1990). Il linguaggio della visione. Bari: Dedalo.

Kim Mintai, Cheon Sanghyun, Kang Youngeun (2018). Use of Electroencephalography (EEG) for the Analysis of Emotional Perception and Fear to Nightscapes. September, pp. I- 15.

Kotowski Krzysztof, Stapor Katarzyna, Leski Jacek, Kotas Marian (2018). Validation of Emotiv EPOC+ for extracting ERP correlates of emotional face processing. In Biocybernetics and Biomedical Engineering, 38(4), pp. 773-78I.

Millennium Ecosystem Assessment (2005). Ecosystems and Human Well-Being: Synthesis. Washington: Island Press.

Posner Jonathan, Russell James A., Peterson Bradley S. (2008). The circumplex model of affect. In Dev Psychopathol, I7(3), pp. 7I5-734.

Schram-Bijkerk Dieneke, Otte Piet, Dirven Liesbet, Breure Anton M. (20 I 8). Indicators to support healthy urban gardening in urban management. In Science of the Total Environment, 621, pp. 863-871.

Tachi Tomohiro (2012). Interactive Freeform Design of Tensegrity. In Hesselgren Lars, Sharma Shrikant, Wallner Johannes, Baldassini Niccolo, Bompas Philippe, Raynaud Jacques (eds.). Proceedings of the Advances in Architectural Geometry Conference. Cham: Springer, pp. 259-268.

Yadava Mahendra, Kumar Pradeep, Saini Rajkumar, Roy Partha Pratim, Prosad Dogra Debi (2017). Analysis of EEG signals and its application to neuromarketing. In Multimedia Tools and Applications, 76 ( | 8), pp. | 9087- |9 | | |.

Yik Michelle, Russell James A., Steiger James H. (20I I). A 12-Point Circumplex Structure of Core Affect. In Emotion, I I (4), pp. 705-731.

\section{Authors}

Fabio Bianconi, Dept. of Civil and Environmental Engineering, University of Perugia, fabio.bianconi@unipg.it

Marco Filippucci, Dept.of Civil and Environmental Engineering, University of Perugia, marco.filippucci@unipg.it,

Marco Seccaroni, Dept. of Civil and Environmental Engineering, University of Perugia, marco.seccaroni@unipg.it 
